LETTERS

\title{
Screening for Intimate Partner Violence at VA EDs: The Time Is Now
}

\author{
Nancy Lutwak, M.D. \\ Department of Emergency Medicine, VA New York Harbor Healthcare System, NYU School of Medicine, New York, NY, USA.
}

$\mathrm{J}$ Gen Intern Med 29(2):279

DOI: $10.1007 / \mathrm{s} 11606-013-2717-5$

(c) Society of General Internal Medicine 2013

To the Editors:- Intimate partner violence is a serious societal problem and contributes significantly to negative health consequences. The recently published editorial by Mitchell D. Feldman focused on this crisis and recommended routine screening by primary care providers. Recent publications have discussed screening for IPV at Veterans Health Administration (VHA) Centers. ${ }^{1-3}$

We agree with Dr. Feldman's recommendations, and also strongly recommend that screening be routinely instituted in all EDs. This is particularly the case at VHA EDs. Women veterans frequently face physical, psychological and social challenges resulting from military sexual trauma. Screening for military sexual trauma is currently performed at VHA clinics, and is currently being introduced as part of ED protocols. ${ }^{1,4}$ Screening for IPV should also be incorporated into VHA ED protocols.

Lifetime exposure to intimate partner violence among women Veterans is estimated at $33 \%$. Intimate partner violence is linked to negative mental health consequences, substance abuse, depression, increased rates of suicide attempts, sleep disturbance and chronic health problems. In 2012, the United States Preventive Services Task Force recommended routine screening of all women of child-bearing age for abuse and intimate partner violence (IPV). The American College of Emergency Physicians subsequently issued a policy that such screening should be performed in emergency department settings. Screening for IPV is also recommended by the National Center of Injury Prevention and Control, as well as the American Medical Association. Screening for IPV should be instituted at VHA EDs nationally, given the scope and negative consequences of this problem.

Corresponding Author: Nancy Lutwak, M.D.; Department of Emergency Medicine, VA New York Harbor Healthcare System, NYU School of Medicine, 423 East 23rd Street, New York, NY 10010, USA (e-mail: nancy.lutwak@gmail.com).

\section{REFERENCES}

1. Feldman MD. Screening for intimate partner violence: the time is now. $J$ Gen Intern Med. 2013. doi:10.1007/s11606-013-2576-0.

2. Sweeney AC, et al. Intimate partner violence: perspectives on universal screening for women in VHA primary care. Women's Health Issues. 2013;23(2):e73-e76.

3. Iverson KM, et al. Clinical utility of an intimate partner violence screening tool for female VHA patients. J Gen Intern Med. 2013;28(10):1288-93. doi:10.1007/s11606-013-2534-x.

4. Lutwak N, Dill C. Military sexual trauma increases risk of post-traumatic stress disorder and depression thereby amplifying the possibility of suicidal ideation and cardiovascular disease. Mil Med. 2013;178(4):35961. doi:10.7205/MILMED-D-12-00427.

Published online November 20, 2013 